\title{
Von Willebrand factor collagen-binding capacity predicts in-hospital mortality in COVID-19 patients: insight from VWF/ADAMTS13 ratio imbalance
}

\author{
Aurélien Philippe ${ }^{1,2} \cdot$ Nicolas Gendron $^{1,2} \cdot$ Olivier Bory $^{3} \cdot$ Agathe Beauvais $^{3} \cdot$ Tristan Mirault $^{4,5}$. \\ Benjamin Planquette ${ }^{1,6} \cdot$ Olivier Sanchez ${ }^{1,6}$. Jean-Luc Diehl ${ }^{1,7} \cdot$ Richard Chocron $^{3,4} \cdot$ David M. Smadja $^{1,2}$
}

Received: 1 April 2021 / Accepted: 3 April 2021 / Published online: 11 May 2021

(C) The Author(s), under exclusive licence to Springer Nature B.V. 2021

\begin{abstract}
Background Microthrombosis is a hallmark of COVID-19. We previously described von willebrand factor (VWF) and their high molecular weight multimers (HMWMs) as potential trigger of microthrombosis.

Objectives Investigate VWF activity with collagen-binding assay and ADAMTS13 in COVID-19.

Methods and results Our study enrolled 77 hospitalized COVID-19 patients including 37 suffering from a non-critical form and 40 with critical form. Plasma levels of VWF collagen-binding ability (VWF:CB) and ADAMTS13 activity (ADAMTS13:Act) were measured in the first 48 hours following admission. VWF:CB was increased in critical (631\% IQR [460-704]) patients compared to non-critical patients (259\% [235-330], $p<0.005)$. VWF:CB was significantly associated $(r=0.564, p<0.001)$ with HMWMs. Moreover, median ADAMTS13:Act was lower in critical (64.8 IU/dL IQR 50.0-77.7) than non-critical patients (85.0 IU/dL IQR 75.8-94.7, $p<0.001$ ), even if no patients displayed majors deficits. VWF:Agto-ADAMTS13:Act ratio was highly associated with VWF:CB $(r=0.916, p<0.001)$. Moreover, VWF:CB level was highly predictive of COVID-19 in-hospital mortality as shown by the ROC curve analysis (AUC $=0.92, p<0.0001$ ) in which we identified a VWF:CB cut-off of $446 \%$ as providing the best predictor sensitivity-specificity balance. We confirmed this cutoff thanks to a Kaplan-Meier estimator analysis (log-rank $p<0.001)$ and a Cox-proportional Hazard model $(\mathrm{HR}=49.1,95 \%$ CI 1.81-1328.2, $p=0.021$ ) adjusted on, BMI, C-reactive protein, and D-dimer levels.

Conclusion VWF:CB levels could summarize both VWF increased levels and hyper-reactivity subsequent to ADAMTS13 overflow and, therefore, be a valuable and easy to perform clinical biomarker of microthrombosis and COVID-19 severity.
\end{abstract}

Keywords COVID-19 $\cdot$ Microthrombosis $\cdot$ Von Willebrand factor $\cdot$ Collagen-binding $\cdot$ Multimers $\cdot$ ADAMTS13 $\cdot$ Mortality

\section{Dear Editor,}

We would like to thank Bhogal et al. [1] for their insightful response to our study demonstrated the importance of von Willebrand factor (VWF) and in particular their high

David M. Smadja

david.smadja@aphp.fr

1 Université de Paris, Innovative Therapies in Haemostasis, INSERM, 75006 Paris, France

2 Hematology Department and Biosurgical Research Lab (Carpentier Foundation), European Georges Pompidou Hospital (HEGP; AP-HP.CUP), 75015 Paris, France

3 Emergency department, European Georges Pompidou Hospital (HEGP; AP-HP.CUP), 75015 Paris, France molecular weight multimers (HMWMs) [2]. They propose perspectives in COVID-19 treatment according to VWF/ ADAMTS13 (a disintegrin and metalloproteinase with a thrombospondin type 1 motif, member 13) axis involvement. VWF and ADAMTS13 form a system known to play

4 Université de Paris, PARCC, INSERM, 75015 Paris, France

5 Vascular Medicine Department, European Georges Pompidou Hospital (HEGP; AP-HP.CUP), 75015 Paris, France

6 Respiratory Medicine Department and Biosurgical Research Lab (Carpentier Foundation), European Georges Pompidou Hospital (HEGP; AP-HP.CUP), 75015 Paris, France

7 Intensive Care Unit and Biosurgical Research Lab (Carpentier Foundation), European Georges Pompidou Hospital (HEGP; AP-HP.CUP), 75015 Paris, France 
a key role in microthrombosis. Indeed, VWF is paramount for both platelet adhesion to subendothelial collagen fibers and platelet aggregation under high shear [3]: VWF is released into the circulation from endothelial cells as hyperactive ultralarge VWF multimers (ULVWFMs). ADAMTS13 controls the breakdown and regulation of ULVWFMs resulting in a balanced distribution between VWF low-, intermediate-, and HMWMs [3]. Microthrombosis, in particular in lungs, is now a hallmark of COVID19. Indeed, significant pulmonary microthrombosis could trigger major gas exchanges defects triggering acute respiratory distress syndrome [4]. As a matter of fact, we previously demonstrated that pulmonary obstruction could lead to right heart ventricle dysfunction linked to COVID19 severity [5]. This microthrombosis is associated to endothelial dysfunction, reflected by increased levels of circulating endothelial cells, plasma angiopoietin-2, and VWF in severe COVID-19 patients [2, 5-7]. Moreover, data on ADAMTS13 activity (ADAMTS13:Act) in severe COVID-19 patients are conflicting, some authors reporting strictly normal [8], slightly decreased [9], or even greatly diminished values [10].

In the present study, we intend to highlight VWF hyperactivity through a rapid and easy-to-perform VWF collagenbinding (VWF:CB) assay. We further explored the cause of the abovementioned VWF hyperactivity in COVID-19, which still remains elusive, through ADAMTS13:Act assessment. In this monocentric cross-sectional study, we explored 77 COVID-19 patients hospitalized including 37 suffering from a non-critical form and 40 with critical form. Patients were all included and COVID-19 severity stratified as previously described [2]. All laboratory assays were performed on samples collected less than $48 \mathrm{~h}$ following patients' hospitalization. Determination of D-dimer and C-reactive protein (CRP) levels, platelet count, VWF:antigen (VWF:Ag), and VWF multimeric profile were achieved as previously described [2]. The VWF:CB was determined using the Asserachrom VWF:CB ELISA kit (Stago, Asnières-surSeine, France). ADAMTS13:Act was assessed using the Technozyme ADAMTS13 activity ELISA (Technoclone, Vienna, Austria). Both ELISA kits were used according to the manufacturer's instructions.

Among the 77 COVID-19 patients hospitalized and studied here, 33 (83\%) critical patients and $25(68 \%)$ noncritical patients were male. Critical patients were not significantly older than non-critical patients (critical: median age of 62 years, interquartile range [IQR] 53-72; non-critical: 63 years IQR $52-72, p=0.85$ ). Conversely, critical patients had significantly higher median body mass index (BMI) than non-critical patients (critical: $28.9 \mathrm{~kg} / \mathrm{m}^{2}$, IQR 26.9-34.9; non-critical: $25.1 \mathrm{~kg} / \mathrm{m}^{2} \mathrm{IQR} 23.6-29.5$, $p=0.005)$. There was no significant difference in median platelet count between critical (307 G/L, IQR 199-375) and non-critical patients (275 G/L IQR 199-375). It is to be noted that we previously described that critical patients displayed higher levels of plasma VWF:Ag associated with a higher proportion of HMWMs compared with non-critical COVID-19 patients [2].

VWF:CB levels on admission were significantly higher in critical (median 631\% IQR [460-704]) than in noncritical (259\% [235-330], $p<0.005$ ) COVID-19 patients (Fig. 1a). Moreover, the VWF:CB-to-VWF:Ag ratio, an indirect and quantitative way to measure VWF multimers size, was still significantly higher in critical (median 1.16 IQR 0.96-1.28) than in non-critical (0.91, IQR 0.85-1.07, $p=0.0001)$ COVID-19 patients, therefore, in favor of VWF hyperactivity in critical patients (Fig. 1b). Consistently, both VWF:CB and the VWF:CB-to-VWF:Ag ratio were strongly positively associated with HMWMs of VWF in our cohort (Spearman correlation coefficient $r=0.564$ and $r=0.731$, respectively, $p<0.0001$ for both) (Fig. 1c and d). Therefore, the VWF:CB assay appears to be an adequate surrogate method to appreciate VWF multimers levels.

Plasma ADAMTS13:Act levels at admission were significantly lower in critical (median 64.8 IU/dL IQR 50.0-77.7) than in non-critical COVID-19 patients (85.0 IU/dL IQR $75.8-94.7, p<0.001$ ) (Fig. 1e). Nevertheless, only 3 (7.5\%) critical patients displayed ADAMTS13:Act levels under normal values (i.e., 40-130 UI/dL), while all non-critical patients showed ADAMTS13:Act levels in the normal range. Moreover, VWF:Ag-to-ADAMTS13:Act ratio is higher in critical (median 8.27 IQR 6.20-11.1) than noncritical patients, (3.46 IQR 2.61-4.30, $p<0.001$ ) supporting a potential saturation of ADAMTS13 in critical COVID-19 patients (Fig. 1f). This result means that for a single unit of plasma ADAMTS13, there was approximately three times more and eight times more circulating VWF:Ag, in noncritical and critical COVID-19 patients, respectively. Notably, the VWF:Ag-to-ADAMTS13:Act ratio was strongly associated with VWF:CB (Spearman correlation coefficient $r=0.916, p<0.001$ ), suggesting that VWF:CB summarize both quantitative (i.e., plasma VWF:Ag increase) and qualitative VWF abnormalities (VWF excess of HMWMs consequential to ADAMTS13 overflow, Fig. 1g).

Finally, we evaluated the discriminatory ability between survivors and non-survivors of VWF:CB using a ROC curve (AUC $=0.92, p<0.0001$, Fig. 2a). Therefore, we estimated a cut-off value to predict in-hospital mortality. Upon admission, a VWF:CB level of $446 \%$ provided an optimal sensitivity-specificity balance, with an excellent sensitivity of $95.2 \%$ (95\% confidence interval [CI] $73.3-99.8 \%$ ) associated with an acceptable specificity of $76.8 \%$ (95\% CI 64.2-85.9\%) regarding COVID-19-related mortality. The ability of this VWF:CB cut-off to predict inhospital mortality was further validated in a Kaplan-Meier 
A

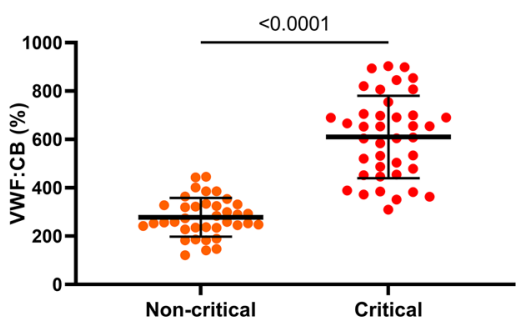

D

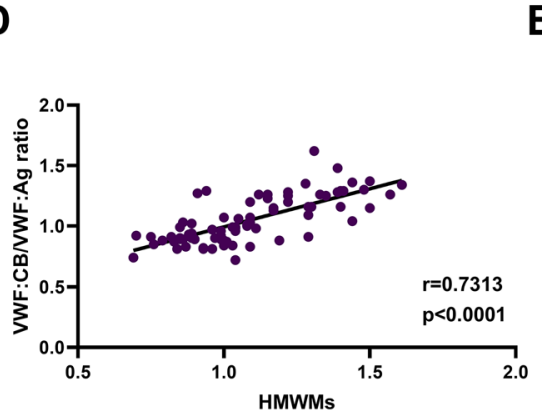

B

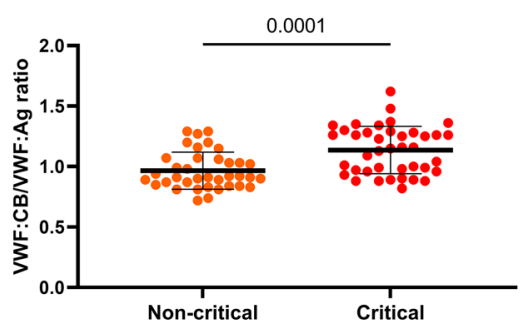

$\mathbf{E}$

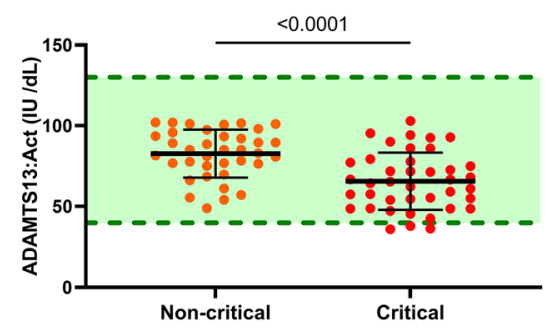

C

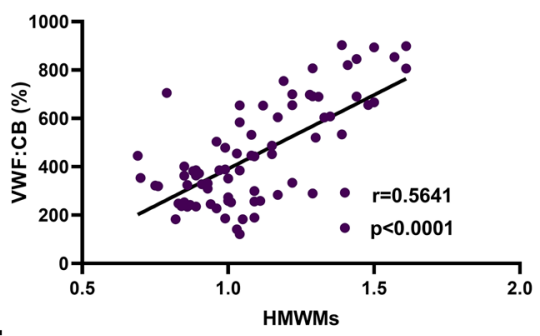

$\mathbf{F}$

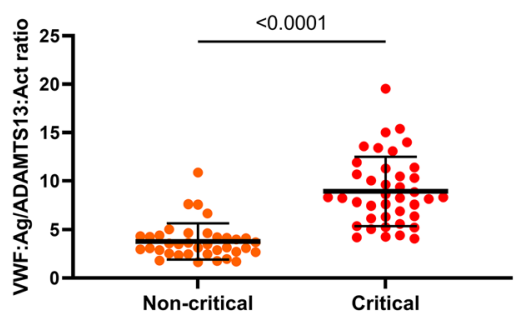

G

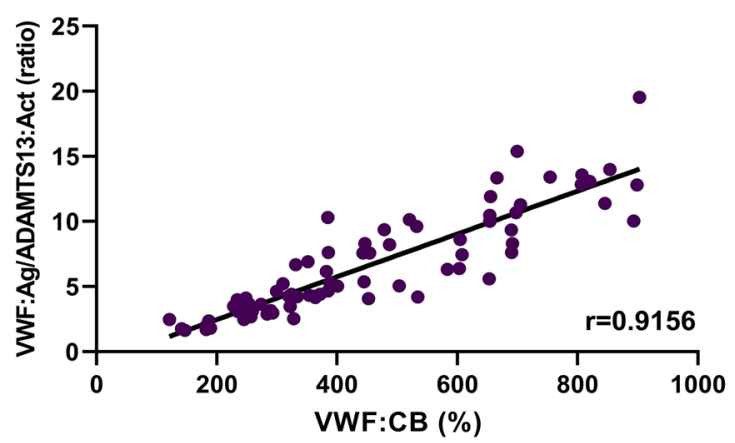

Fig. 1 Levels and association of von Willebrand factorADAMTS13 system-related biomarkers according to critical and non-critical COVID-19 patients. Data points indicate individual measurements, whereas horizontal bars represent the means with standard deviations. Green-shaded area indicate the normal ranges of values. For comparison between severity groups, $p$ value comes from the Mann-Whitney test. For association between two variables in the whole cohort, $\mathrm{p}$ value and correlation coefficient ( $\mathrm{r}$ ) comes from the Spearman correlation and the solid black line was obtained through simple linear regression. Plasma levels of von Willebrand factor:collagen binding (VWF:CB) (a) and VWF:CB-toVWF:antigen (VWF:Ag) ratio (b). Association between VWF high molecular weight multimers (HMWMs) proportion and VWF:CB (c) or VWF HMWMs and VWF:CB-to-VWF:Ag ratio (d). Plasma levels of ADAMTS13 activity (ADAMTS13:Act) (e) and VWF:Ag-toADAMTS13 ratio (f). Association between VWF:Ag-to-ADAMTS13 ratio and VWF:CB (g) estimator ( $p<0.001$, Fig. 2b) and a Cox-proportional hazard analysis adjusted for BMI, D-dimer, and CRP (Hazard ratio [HR] 49.1, 95\% CI 1.81-1328.2, $p=0.021$, Fig. 2c).

Our study highlights increased circulating hyperactive VWF and ADAMTS13 overflow in COVID-19 severity. This imbalance could be at the origin of microthrombosis. Moreover, VWF:CB appears to sum up VWF global thrombogenic impact and is a powerful predictor of COVID-19-related mortality which reinforces this hypothesis.

VWF:CB assay is initially a diagnostic test that quantifies the binding capacity of VWF-A1 and -A3 domain to collagen binding, the main subendothelial matrix component in order to improve the diagnosis and differentiation of qualitative variant of von Willebrand disease, the most common inherited bleeding disorder [3]. VWF activity is commonly assessed using VWF ristocetin cofactor assays, which evaluate the binding between the VWF-A1 domains to platelet membrane receptor GPIb. VWF:CB activity is a more sensitive test exploring HMWMs of VWF abnormalities [11]. Consistently, we observed a marked increase of VWF:CB in critical COVID-19 patients, even after normalization to VWF:Ag levels, confirming an intrinsic VWF hyperactivity, previously described using HMWMs evaluation [2]. VWF multimers quantification is a complex and time-consuming 
A

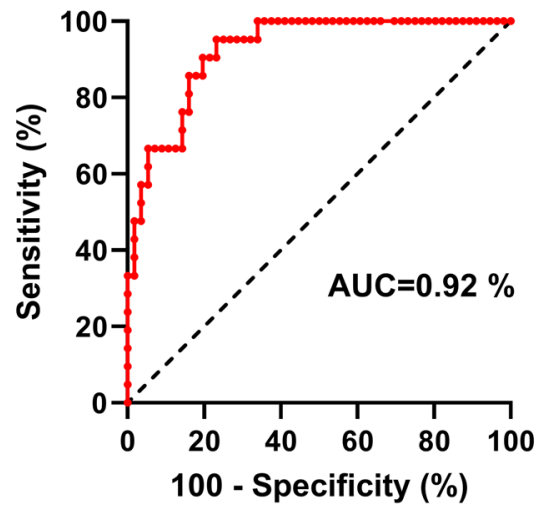

B
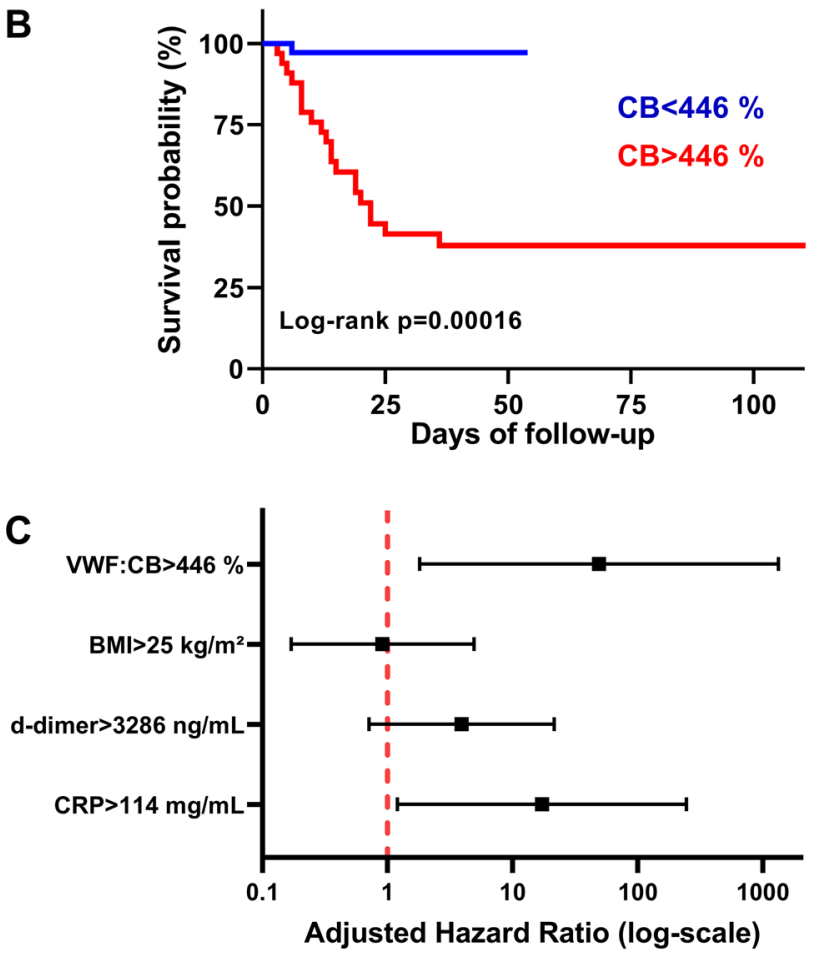

Fig. 2 Association between von Willebrand factor collagen-binding admission level and in-hospital mortality in COVID-19 patients. a Receiver operating curves evaluating von Willebrand factor collagen-binding (VWF: CB) ability to predict in-hospital-mortality estimated by the area under the curve (AUC) value. The diagonal black-dotted segment is the reference line. b Survival curves according to VWF:CB level using a Kaplan-Meier estimator. Data are shown for patients with low VWF:CB $(<446 \%)$ and high von VWF:CB (>446\%). Survival curves are compared using the logrank test. $\mathbf{c}$ Forest plot showing the Cox-proportional hazards model for VWF:CB adjusted for age, body mass index (BMI), D-dimer, and C-reactive protein (CRP). For each variable, black squares represent hazard ratios (HR) and solid black lines represent HR 95\% confidence intervals

method. Thus, VWF:CB could represent a valuable, easy-toperform assay to highlight VWF hyperactivity in COVID-19.

In addition, we found lower ADAMTS13:Act in critical COVID-19 patients than in non-critical ones even if only few patients present values above normal, a finding consistent with some previous reports [9]. Severe deficiency in ADAMTS13 (activity $<10 \mathrm{IU} / \mathrm{dL}$ ) is a signature for thrombotic thrombocytopenic purpura (TTP) diagnosis, a life-threatening thrombotic condition caused by the subsequent accumulation of hyperactive HMWMs causing disseminated thrombi formation occluding arterioles and capillaries [12]. Reports of small platelets-rich thrombi within the microvasculature of SARS-CoV-2-infected patients [13] makes it tempting to draw a parallel between TTP and COVID-19 associated coagulopathy. However, the normal platelet count in nearly all COVID-19 patients in our cohort associated with the scarcity of ADAMTS13:Act true deficits make this hypothesis difficult to accept. Thus, more than a decrease in ADAMTS13:Act levels, we observed a major discrepancy between ADAMTS13:Act and VWF:Ag levels in critical patients, suggesting an overflow of ADAMTS13 capacity. Although TTP arises from severe decreased in ADAMTS13:Act, thrombotic events have been associated to slight decrease if associated with a major increase of circulating VWF level, in particular in myocardial infarction, ischemic stroke, and cancer-associated venous thrombosis [14]. Moreover, Warlo et al. demonstrated a correlation between increased VWF:Ag-to-ADAMTS13:Act ratio and high residual platelet reactivity despite aspirin treatment, suggesting that imbalance in VWF-ADAMTS13 system could also promote arise of hyper-reactive platelets [14]. The mechanisms underlying altered VWF:Ag-to-ADAMTS 13 ratio in COVID-19 is still unknown. ADAMTS13 could be consumed at a higher rate due to increased VWF processing, and/or inflammatory cytokines could suppress ADAMTS13 biosynthesis in the liver [15].

All in all, VWF:CB is significantly associated to VWF:Ag-to-ADAMTS13 ratio and HMWMs of VWF. VWF:CB could summarize both VWF qualitative and quantitative anomalies in COVID-19 and therefore be a valuable, easy-to-perform, mirror of VWF global thrombotic involvement in microthrombosis formation. Our results not only highlight the potential for plasma VWF:CB to discriminate COVID-19 patient's severity and/or in-hospital mortality but also may help targeting patients for new therapeutic approaches such as (i) recombinant ADAMTS13, (ii) caplacizumab (an anti-von Willebrand factor humanized singlevariable-domain immunoglobulin targeting the A1 domain of von Willebrand factor), (iii) $\mathrm{N}$-acetyl cysteine known to reduce the size of VWF multimers.

Funding This work has been funded with grants from French national agency for research ANR SARCODO (Fondation de France) and Mécénat Covid AP-HP. The study was performed in accordance with the Declaration of Helsinki. All patients provided written informed consent before enrollment (CPP 2020-04-048/2020-A01048$31 / 20.04 .21 .49318$ ). The datasets generated during and/or analyzed 
during the current study are available from the corresponding author on reasonable request.

\section{Declarations}

Conflict of interest All author declares that they have no conflict of interest.

\section{References}

1. Bhogal P, Paul G, Collins O (2021) Letter in response to: circulating Von Willebrand factor and high molecular weight multimers as markers of endothelial injury predict COVID-19 in-hospital mortality. Angiogenesis. https://doi.org/10.1007/ s10456-020-09762-6

2. Philippe A, Chocron R, Gendron N et al (2021) Circulating Von Willebrand factor and high molecular weight multimers as markers of endothelial injury predict COVID-19 in-hospital mortality. Angiogenesis. https://doi.org/10.1007/s10456-020-09762-6

3. Lillicrap D (2013) Von Willebrand disease: advances in pathogenetic understanding, diagnosis, and therapy. Hematol Am Soc Hematol Educ Program 2013:254-260

4. Diehl J-L, Peron N, Chocron R et al (2020) Respiratory mechanics and gas exchanges in the early course of COVID-19 ARDS: a hypothesis-generating study. Ann Intensive Care 10:95

5. Goudot G, Chocron R, Augy JL et al (2020) Predictive factor for COVID-19 worsening: insights for high-sensitivity troponin and D-Dimer and correlation with right ventricular afterload. Front Med Front. https://doi.org/10.3389/fmed.2020.586307

6. Smadja DM, Guerin CL, Chocron R et al (2020) Angiopoietin-2 as a marker of endothelial activation is a good predictor factor for intensive care unit admission of COVID-19 patients. Angiogenesis 23(4):611-620
7. Khider L, Gendron N, Goudot $\mathrm{G}$ et al (2020) Curative anticoagulation prevents endothelial lesion in COVID-19 patients. J Thromb Haemost JTH 18(9):2391-2399

8. Escher R, Breakey N, Lämmle B (2020) ADAMTS13 activity, von Willebrand factor, factor VIII and D-dimers in COVID-19 inpatients. Thromb Res 192:174-175

9. Mancini I, Baronciani L, Artoni A et al (2021) The ADAMTS13von Willebrand factor axis in COVID-19 patients. J Thromb Haemost JTH 19:513-521

10. Martinelli N, Montagnana M, Pizzolo F et al (2020) A relative ADAMTS13 deficiency supports the presence of a secondary microangiopathy in COVID 19. Thromb Res 193:170-172

11. Favaloro EJ, Pasalic L, Curnow J (2016) Laboratory tests used to help diagnose von Willebrand disease: an update. Pathology (Phila) 48:303-318

12. Scully M, Cataland S, Coppo P et al (2017) Consensus on the standardization of terminology in thrombotic thrombocytopenic purpura and related thrombotic microangiopathies. J Thromb Haemost JTH 15:312-322

13. Fox SE, Akmatbekov A, Harbert JL et al (2020) Pulmonary and cardiac pathology in African American patients with COVID19: an autopsy series from New Orleans. Lancet Respir Med 8:681-686

14. Warlo EMK, Pettersen AÅR, Arnesen H et al (2017) vWF/ ADAMTS13 is associated with on-aspirin residual platelet reactivity and clinical outcome in patients with stable coronary artery disease. Thromb J 15:28

15. Zheng X, Majerus EM, Sadler JE (2002) ADAMTS13 and TTP. Curr Opin Hematol 9:389-394

Publisher's Note Springer Nature remains neutral with regard to jurisdictional claims in published maps and institutional affiliations. 